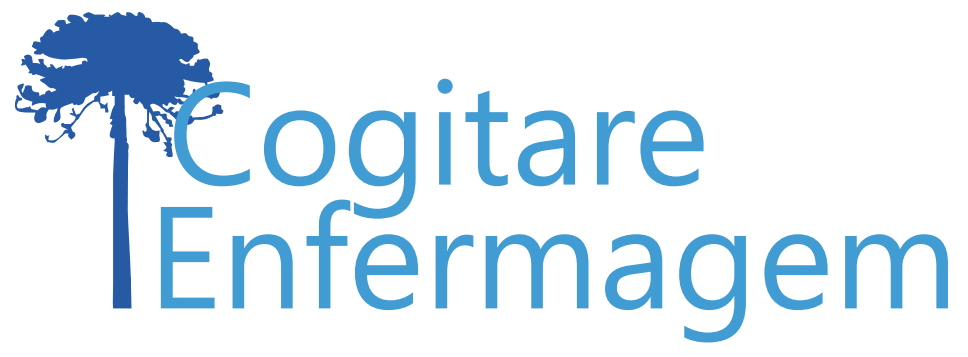

\title{
CUIDADOS DE ENFERMAGEM NO ATENDIMENTO ÀS VÍTIMAS DE PICADAS ESCORPIÔNICAS NA ATENÇÃO PRIMÁRIA À SAÚDE
}

Edjane Xavier dos Santos Silva ${ }^{1}$, Willames da Silva², Jorge Luiz Costa da Fonseca ${ }^{3}$, Salmana Rianne Pereira Alves ${ }^{4}$, Daiane Medeiros da Silva ${ }^{5}$, Jordana de Almeida Nogueira ${ }^{6}$, Débora Raquel Soares Guedes Trigueiro ${ }^{7}$

\section{RESUMO}

Objetivo: avaliar conhecimentos e práticas dos profissionais de enfermagem na atenção primária. Método: estudo exploratório-descritivo, com abordagem qualitativa, desenvolvido de fevereiro a março de 2018, com 20 enfermeiros de Unidades de Saúde da Família, da Paraíba, Brasil, por meio de entrevista semiestruturada, submetida à técnica de análise temática.

Resultados: verificou-se que os enfermeiros não desenvolvem uma assistência sistematizada, mas pontual, com base nos sinais e sintomas do quadro leve, e transferem ao serviço de referência a responsabilidade pelo atendimento ao caso, por desconhecimento das condutas estabelecidas para o acidentado. Ademais, 19 enfermeiros não realizam ações educativas para a prevenção dos acidentes e repassam a responsabilidade ao sistema de vigilância em saúde.

Conclusão: são necessárias a implementação de programas de treinamentos, a elaboração de Procedimento Operacional Padrão e a realização de educação em saúde para os profissionais, a fim de melhorar a assistência às vítimas de picadas escorpiônicas.

DESCRITORES: Picadas de Escorpião; Assistência à Saúde; Enfermagem; Estratégia Saúde da Família; Atenção Primária à Saúde.

\section{COMO REFERENCIAR ESTE ARTIGO:}

Silva EX dos S, Silva W da, Fonseca JLC da, Alves SRP, Silva DM da, Nogueira J de A, Trigueiro DRSG. Cuidados de enfermagem no atendimento às vítimas de picadas escorpiônicas na atenção primária à saúde. Cogitare enferm. [Internet]. 2020 [acesso em "colocar data de acesso, dia, mês abreviado e ano"]; 25. Disponível em: http://dx.doi.org/10.5380/ce.v25i0.67322.

Este obra está licenciado com uma Licença Creative Commons Atribuição 4.0 Internacional.

${ }^{1}$ Enfermeira. Pós-Graduanda em Saúde da Família. Enfermeira Assistencial no Centro de Saúde Dr. José Dustan Carvalho Soares. Recife, PE, Brasil. (2)

${ }^{2}$ Discente de Enfermagem. Faculdade de Enfermagem Nova Esperança. João Pessoa, PB, Brasil. $\bigcirc$

${ }^{3}$ Médico. Mestrando em Saúde da Família. Faculdade de Enfermagem Nova Esperança. João Pessoa, PB, Brasil. 9

${ }^{4}$ Enfermeira. Mestre em Saúde da Família. Docente de Enfermagem da Faculdade de Enfermagem Nova Esperança. João Pessoa, PB, Brasil. 1

${ }^{5}$ Enfermeira. Doutora em Enfermagem. Docente da Faculdade de Enfermagem Nova Esperança. João Pessoa, PB, Brasil. 9 ${ }^{6}$ Enfermeira. Doutora em Enfermagem e Saúde Pública. Docente de Enfermagem da Universidade Federal da Paraíba. João Pessoa, PB, Brasil. 1

${ }^{7}$ Enfermeira. Doutora em Enfermagem. Docente de Enfermagem da Faculdade de Enfermagem Nova Esperança. João Pessoa, PB, Brasil. 


\title{
NURSING CARE ACTIONS IN SERVING VICTIMS OF SCORPION STINGS IN PRIMARY HEALTH CARE
}

\begin{abstract}
Objective: To evaluate the knowledge and practices of nursing professionals in primary care. Method: An exploratory-descriptive study with a qualitative approach developed from February to March 2018 with 20 nurses from Family Health Units in Paraiba, Brazil, through semi-structured interviews, submitted to the thematic analysis technique.

Results: It was verified that the nurses do not develop systematized care, but a punctual one, based on the signs and symptoms of the mild condition, and transfer the responsibility for handling the case to the reference service, due to lack of knowledge of the guideline established for the victim. In addition, 19 nurses do not carry out educational actions to prevent accidents and pass on the responsibility to the health surveillance system.

Conclusion: The implementation of training programs, the elaboration of the Standard Operating Procedure, and the provision of health education for the professionals are necessary in order to improve the care provided to victims of scorpion bites.
\end{abstract}

DESCRIPTORS: Scorpion stings; Health care; Nursing; Family Health Strategy; Primary Health Care.

\section{CUIDADOS DE ENFERMERÍA PROVISTOS A VÍCTIMAS DE PICADURAS DE ESCORPIÓN EN LOS SERVICIOS DE ATENCIÓN PRIMARIA DE LA SALUD}

\section{RESUMEN:}

Objetivo: evaluar los conocimientos y las prácticas de los profesionales de enfermería en la atención primaria.

Método: estudio exploratorio-descriptivo con enfoque cualitativo, desarrollado de febrero a marzo de 2018 con 20 enfermeros de Unidades de Salud de la Familia de Paraíba, Brasil, por medio de entrevistas semiestructuradas, sometidas a la técnica del análisis temático.

Resultados: se verificó que los enfermeros no ofrecen asistencia sistematizada, sino más bien puntual, en función de las señales y de los síntomas del cuadro leve, y que transfieren al servicio de referencia la responsabilidad de manejar el caso por desconocer las conductas establecidas para atender a la víctima. Además, 19 enfermeros no realizan ninguna acción educativa para prevenir los accidentes, además de transferir la responsabilidad al sistema de vigilancia de la salud.

Conclusión: es necesario implementar programas de capacitación, elaborar un Procedimiento Operativo Estándar, y realizar acciones de educación en salud para los profesionales, a fin de mejorar la asistencia que se presta a las víctimas de picaduras de escorpión.

DESCRIPTORES: Picaduras de escorpión; Asistencia a la Salud; Enfermería; Estrategia de Salude de la Familia; Atención Primaria de la Salud. 
O ser humano sempre sofreu com ataques de animais peçonhentos. A preocupação com esses animais se dá devido ao veneno expelido, que pode causar sérios problemas à saúde de suas vítimas, como também gerar prejuízos financeiros à Saúde Pública(1).

Dentre os diversos tipos de animais peçonhentos, destaca-se o escorpião, que tem apresentado uma incidência preocupante em locais urbanos, em decorrência, muitas vezes, do desmatamento. Essa ação altera o ambiente natural desses seres, diminuindo os recursos para sua sobrevivência e aumentando a sua invasão em residências, terrenos baldios e em áreas de construção(2).

Acidentes por escorpiões constituem um atual problema de saúde pública no Brasil, e a redução do número de ocorrências deve ser feita de forma preventiva. A maneira mais eficaz para combater essa problemática é o seu conhecimento clínico e epidemiológico ${ }^{(2)}$.

Nesse sentido, a implantação da notificação dos acidentes escorpiônicos no Brasil em 1988 e a melhora no registro desse agravo contribuíram para a elevação na taxa de incidência. Segundo dados do Ministério da Saúde, no Brasil foram registrados 951.727 casos de acidentes por escorpiões, no período entre 2000 e $2017^{(2,3)}$.

É necessário controlar o crescimento das populações desses animais, dado o risco que eles representam para a saúde humana. Como medida para conter esse avanço, o Governo Brasileiro criou a Portaria $n^{\circ}$ 1.172, de 15 de junho de 2004, que em seu artigo $3^{\circ}$, inciso 10, estabelece ao município o registro, a captura, a apreensão e a eliminação de animais que representem risco à saúde do homem, cabendo ao Estado a supervisão, acompanhamento e orientação dessas ações ${ }^{(4)}$.

Em nível municipal, segundo o Programa Nacional de Atenção Básica (PNAB), uma das características das equipes é programar e implementar ações de saúde, de acordo com a necessidade da população, priorizando intervenções clínicas e sanitárias nos problemas de saúde, conforme critérios de frequência, risco, vulnerabilidade e resiliência(5).

Dessa forma, os profissionais de enfermagem devem estar capacitados para prestar assistência adequada à vítima de picada de escorpião que ocorra na sua área de atuação, uma vez que o acidentado poderá acessar o serviço de saúde mais próximo e este deve ser resolutivo em sua demanda ao seguir o manual de diagnóstico e tratamento de acidentes por animais peçonhentos ${ }^{(6)}$. Ressalta-se que 0 atendimento a essas pessoas requer qualidade e agilidade, o que contribui para a exatidão no diagnóstico e, quando necessário, o encaminhamento das vítimas às unidades de saúde especializadas.

Frente à problemática, entende-se que a avaliação do conhecimento e das práticas realizadas pelos profissionais de enfermagem diante de um acidente escorpiônico permitirá qualificar o serviço de assistência terapêutica e preventiva realizados atualmente. Possíveis deficiências encontradas neste processo servirão de subsídio para a implementação de ações de melhoria da assistência à saúde das pessoas picadas por escorpião. Desse modo, este estudo objetivou avaliar conhecimentos e práticas dos profissionais de enfermagem na atenção primária acerca de acidentes escorpiônicos.

\section{MÉTODO}

Pesquisa de caráter exploratório e descritivo com abordagem qualitativa, desenvolvido nas Unidades de Saúde da Família (USF) pertencentes ao Distrito Sanitário III (DS III), situadas no município de João Pessoa, Paraíba, Brasil.

A população do estudo foi de 60 enfermeiros, destes participaram 20 enfermeiros, 
obedecendo à técnica de saturação de respostas. Para a coleta das informações, foi elaborado pelos autores um roteiro de entrevista semiestruturado, individual, com gravação MP3, contendo quesitos pertinentes aos dados de caracterização do acidente, quadro clínico decorrente da picada, assistência ofertada ao usuário, ações que não devem ser realizadas frente à ocorrência, e medidas preventivas na comunidade exposta ao agravo.

Os dados foram coletados durante os meses de fevereiro e março de 2018, conforme o funcionamento da instituição, nos turnos manhã e tarde, de acordo com a disponibili $\neg$ dade dos participantes e após a leitura e a assinatura do TCLE.

As gravações foram transcritas de forma integral e submetidas à técnica de análise de conteúdo, modalidade temática ${ }^{(7)}$. Para a interpretação dos dados, os núcleos de sentidos foram organizados em categorias. Para preservar seu anonimato, os profissionais foram identificados pela letra $\mathrm{E}$ de enfermeiro, seguido de números cardinais crescentes relativos à ordem das entrevistas.

A pesquisa foi aprovada pelo Comitê de Ética em Pesquisa da Faculdade de Enfermagem Nova Esperança sob o Parecer $n^{\circ} 2.866 .645$, e respeitou os aspectos éticos contidos na Resolução n ${ }^{\circ} 66 / 12$ do Conselho Nacional de Saúde ${ }^{(8)}$.

\section{RESULTADOS}

Após leitura do material empírico, por similaridade de conteúdo, emergiram quatro categorias que proporcionaram o entendimento da assistência de enfermagem dispensada em nível da APS no DS III do município investigado.

\section{Categoria 1 - Ações pontuais sem aprofundamento dos profissionais no atendimento inicial}

Inicialmente, o profissional de saúde foi questionado sobre quais informações são coletadas quando recebe o acidentado em sua unidade. Observou-se que o enfermeiro(a) de forma assertiva identifica algumas ações pontuais, mas sem aprofundamento e completude das competências, tais como: tipo de escorpião, se a vítima capturou o animal, data do acidente, quadro clínico, área da picada, procedimento que o usuário teve no local do acidente e encaminhamento ao serviço de referência do município - Centro de Assistência Toxicológica (Ceatox).

Eu geralmente pergunto sobre o escorpião, como era, se conseguiu capturar, aí depois vou para olhar o local e os sintomas que estava sentindo, dependendo dos sintomas, já encaminho para o Ceatox [...]. (E.01)

Primeiro eu pergunto quando foi a picada, o dia que foi, se ele sabe o tipo do escorpião e o que ele fez no local da picada [...]. (E.03)

Em seguida, o profissional de saúde foi indagado acerca dos sintomas que o usuário pode apresentar após ser picado por um escorpião, revelando-se que a maioria dos respondentes ressaltaram dor intensa e hiperemia no local do acidente. Além desses sintomas, foram mencionados edema local, rubor, febre, parestesia, tontura, ardência, dormência, náusea, vômito e cefaleia.

Edema, rubor, dor de cabeça, náusea, dor local. A dor é a mais forte que tem, o principal deles é a dor [...]. (E.02)

[...] Uma dor bem forte e formigamento nos membros [...]. (E.10)

Ardência e dor no local, vermelhidão e formigamento. Pergunto se tem náusea, vômito e 


\section{Categoria 2 - Cuidado fragmentado e transferência de responsabilidade ao serviço de referência}

A seguir, os enfermeiros foram indagados sobre quais ações de saúde deveriam ser ofertadas ao usuário picado por escorpião. Dentre as apontadas, estão a prescrição de analgésico ou antialérgico, orientação ao acidentado para tomar analgésico, fazer compressa fria ou lavar o local da picada com água e sabão e encaminhar para o Ceatox.

[...] Eu oriento a compressa fria, encaminho e que ele tome algum tipo de analgésico. (E.03)

Na parte da enfermagem, a gente só orienta com relação à limpeza ou encaminha para o Ceatox [...]. (E.07)

Na unidade, a gente encaminha para o Hospital Universitário onde fica o Ceatox, mas a gente pode fazer um antialérgico e pode fazer um analgésico [...]. (E.10)

Concomitantemente, uma parcela significativa dos profissionais desconhece a assistência que deve ser ofertada ao usuário picado por escorpião, como também nega haver essa atenção na ESF.

Falando sério, eu desconheço o tratamento, o que é que o Ceatox faz. Eu desconheço [...]. (E.16)

Até onde eu sei, é o analgésico mesmo, se for o escorpião normal; se for o de barriga escura, confesso que não conheço muito essa parte. Eu não tenho essa experiência para te dizer [...]. (E.17)

Aqui não tem tratamento para esse acidente [...]. (E.20)

\section{Categoria 3 - Cuidado inicial da comunidade mediante conhecimentos oriundos da cultura popular}

Foram elencados pelos enfermeiros alguns procedimentos que não devem ser feitos diante de um acidente escorpiônico, mas que a comunidade costuma realizar, como colocar alho, álcool, curativo, anti-inflamatório, pó de café, tempero, creme dental, pomada, manteiga, terra, chá, vinagre, espremer, cortar ou chupar o local da picada e fazer curativo.

[...] A gente vê muito o pessoal espremendo o local da picada [...] O povo faz uns temperos e bota, que não deve ser feito [...]. (E.14)

O que não deve fazer é fechar, tampar o local, colocar algum tipo de faixa [...]. (E.15)

Cortar, chupar, o povo tem essa mania [...]. (E.17)

Não colocar alho, nem álcool, nem curativo, ou creme dental, umas pomadas que tenham em casa, tomar anti-inflamatório que também tem em casa [...]. (E.20)

\section{Categoria 4 - Prevenção sem prevenir - intersetorialidade para o controle ambiental apenas quando há ocorrência}

Finalizando o questionamento, o profissional de saúde citou quais medidas de prevenção toma frente à comunidade exposta a acidentes envolvendo escorpiões e a forma como essas ações são realizadas. Constatou-se que o enfermeiro aciona o Centro de Controle de Zoonoses (CCZ), a Vigilância Ambiental, a Vigilância Epidemiológica, o Ceatox e o órgão responsável por dedetização; faz parceria com os Agentes de Combate a Endemias (ACE) e os Agentes Comunitários de Saúde (ACS) para que estes profissionais 
verifiquem as condições de higiene no entorno das moradias e realizem educação sanitária.

Eu acho que eu ligaria para o Ceatox [...]. (E.01)

Eu acho que acionar o órgão competente para fazer um trabalho na área. Seria um trabalho de dedetização [...]. (E.02)

[...] A gente entra em contato com a Vigilância Epidemiológica para fazer as visitas nas casas e orientar a comunidade [...]. (E.06)

[...] Uma das coisas que a gente faz é contato com a rede de Zoonoses. [...] é o Centro de Zoonoses que faz esse trabalho junto com os agentes de endemias. (E.16)

Dos profissionais entrevistados, dois afirmaram somente realizar medidas de prevenção na comunidade após receberem casos de acidentes com escorpião nas unidades de saúde em que trabalham.

[...] Eu geralmente não faço isso como prevenção, só quando a coisa ocorre geralmente [...]. (E.12)

[...] Não tem rotina de estar fazendo esse repasse para a população. A gente só atenta de orientar quando tem os casos. (E.19)

Os acidentes por escorpião acontecem em todo o território nacional e o aumento da taxa de incidência anual está relacionado a fatores como a destruição do habitat natural desses animais, a falta de infraestrutura e o crescimento desordenado dos grandes centros urbanos, que resulta em moradias precárias, falta de saneamento básico, sem coleta de lixo, o que facilita a presença e proliferação desses artrópodes no ambiente domiciliar ${ }^{(9)}$.

A gravidade dos acidentes provocados por escorpiões varia conforme o tipo e o tamanho do animal, o local da picada, a idade da vítima, a quantidade de veneno injetado e o tempo maior do que três horas decorrido entre a picada e o tratamento ${ }^{(10)}$.

É necessário identificar o tipo do escorpião pelo qual a vítima foi picada, visto que os acidentes graves e fatais estão associados à espécie T. serrulatus, enquanto que o veneno do T. stigmurus apresenta um grau menor de toxicidade, provocando nas vítimas manifestações clínicas leves e localizadas ${ }^{(1,10)}$. É relevante também saber se a vítima capturou o animal, uma vez que essa captura contribui para o controle da população de escorpiões para a produção do antiveneno e para a identificação de espécies não registradas na região ${ }^{(1,4)}$.

A apuração da data do acidente escorpiônico foi descrita em pesquisas ${ }^{(1,11)}$ que reforçam a importância de se obter essa informação, pois é fundamental para o melhor prognóstico do acidente com escorpião, uma vez que a ação da toxina ocorre em poucos minutos após a picada, agravando o quadro clínico do paciente com o passar do tempo. É crucial que o quadro clínico seja analisado de maneira criteriosa, pois os sintomas determinam a classificação do caso e podem indicar ou não o uso do soro(11).

Estudos ${ }^{(12,13)}$ mostram que complicações e possíveis sequelas são maiores se o local do acidente for próximo de órgãos vitais. Ademais, é importante saber do paciente quais foram as condutas que ele teve no local da picada, haja visto que medidas como colocar ervas, pó de café, entre outras, afetam o tratamento, elevando a possibilidade de infecções.

Verificou-se que nenhum entrevistado abordou a idade da vítima, no momento em que 0 acidentado chega à unidade de saúde. Fato preocupante, pois pesquisas ${ }^{(10,14)}$ revelam que o risco de morbidade e mortalidade é maior em crianças menores de 7 anos e 
em idosos, já que seus sistemas imunológicos estão em desenvolvimento ou em declínio, respectivamente.

Sobre o quadro clínico dos acidentes escorpiônicos, os sintomas como dor local, edema, hiperemia e parestesia foram mencionados pelos enfermeiros e, de acordo com estudos $^{(13,15)}$, são classificados como leves. A maioria dos acidentes possui esse nível de classificação e a dor constitui o principal sinal clínico, estando presente em todos os casos.

A sintomatologia descrita corresponde, em sua maioria, aos casos leves, tendo os sintomas moderados sido citados por apenas dois profissionais. Nenhum sintoma grave foi mencionado. Este resultado pode refletir a falta de conhecimento dos enfermeiros acerca do assunto, indicando uma possível carência de capacitação. É indispensável que o profissional de enfermagem saiba identificar os sinais e sintomas locais e sistêmicos, como também as complicações dos acidentes escorpiônicos, no atendimento pré-hospitalar. Isso favorece a rapidez da chegada da vítima à unidade de referência, onde receberá o atendimento seguro(16).

Em relação ao cuidado prestado aos acidentados, observou-se que uma das condutas realizadas pelos enfermeiros é fazer compressa gelada no local da picada. Segundo pesquisa(17), este procedimento é contraindicado, pois o uso de compressas com gelo ou água gelada acentua o quadro de dor, além de serem ineficazes e aumentarem o risco de complicações.

tratamento relatado pelos entrevistados refere-se aos casos leves e consiste no controle da dor. Para estes casos, indica-se a utilização de analgésicos sistêmicos, como a dipirona, e anestésicos sem vasoconstritor, como a lidocaína (a 2\%), que deve ser injetada no local da picada ou sob forma de bloqueio ${ }^{(14)}$.

Nenhum profissional indicou o tratamento correto para os casos moderados e graves, quando era esperado o apontamento do uso de sorologia. Mesmo que esta terapia não seja disponibilizada pela ESF, é importante que o profissional de enfermagem tenha conhecimento desse recurso terapêutico.

Uma possível explicação para o desconhecimento desses profissionais sobre a assistência que deve ser ofertada ao acidentado pode ser vista numa pesquisa realizada no município de Cuiabá-MT(18), que mostra que o profissional de saúde tem pouco conhecimento sobre acidentes causados por animais peçonhentos, resultado da carência de informações recebidas durante a formação acadêmica e da ausência de qualificações para os profissionais abordando a temática.

Diante do exposto, há necessidade de um planejamento efetivo das atividades de Educação Permanente em Saúde (EPS) nas USF, inserindo os profissionais de enfermagem nestas ações, a fim de prepará-los para o atendimento adequado às vítimas. Ainda, é crucial que as condutas de diagnósticos e tratamento dos acidentados tenham uma padronização atualizada e estejam sempre disponíveis, uma vez que os profissionais de saúde se deparam regularmente com vítimas deste tipo de acidente ${ }^{(18)}$.

Quanto às ações inadequadas frente a um acidente escorpiônico, os enfermeiros elencaram algumas condutas oriundas da cultura popular que podem prejudicar 0 diagnóstico e o tratamento correto. Destaca-se que o conhecimento popular tem seu valor, porém, em algumas situações, torna-se equivocado, como exemplo, fazer torniquete em caso de acidentes com animais peçonhentos, cujo procedimento agrava a ação proteolítica do veneno, aumentando o risco de infecção(19).

No que se refere às medidas preventivas dos acidentes, observou-se que os profissionais não têm uma rotina padronizada e acionam outros órgãos públicos para efetuar as ações. Entre estes, o CCZ foi um dos centros municipais ligado à Secretaria de Saúde solicitado. Este órgão é responsável pela prevenção e controle de zoonoses e de acidentes causados por animais peçonhentos e venenosos, de relevância para a saúde pública ${ }^{(4,20)}$. 
Além dos órgãos componentes do sistema de vigilância em saúde, é importante enfatizar a atuação de outros atores no combate aos escorpiões, como os ACS e os ACE, que desenvolvem um trabalho importante dentro do SUS. Ambos realizam suas atividades junto à comunidade a fim de facilitar o acesso aos serviços de saúde e prevenir doenças ${ }^{(5,21)}$.

Vale destacar que não é competência da vigilância epidemiológica, nem do Ceatox, realizar medidas preventivas in loco contra animais peçonhentos. A vigilância epidemiológica tem como função, dentre outras: coletar e inserir os dados nos sistemas de informação em saúde; analisar e interpretar os dados processados; e divulgar o resultado obtido. Ressaltase que é a partir dos resultados que os órgãos competentes fazem a atuação direta no combate aos agravos. Já o Ceatox tem como finalidade fornecer informações ao público sobre intoxicações e envenenamentos, através de atendimento telefônico, e prestar assistência às pessoas expostas e/ou intoxicadas, a fim de reduzir a morbimortalidade ${ }^{(22,23)}$.

Sabe-se que a dedetização para o controle de escorpiões é contraindicada, devido à falta de estudos que comprovem a eficácia do tratamento químico. O que torna esse método ineficaz é que os escorpiões conseguem permanecer com seus estigmas pulmonares fechados por um longo período, como também podem ficar meses sem se movimentar. Ademais, a aplicação de produtos químicos nos habitats em que esses animais vivem pode causar o seu desalojamento, aumentando o risco de acidentes ${ }^{(4,19)}$.

Foi possível constatar que apenas um enfermeiro informou realizar medidas educativas para a população, enquanto os demais transferem a responsabilidade das ações de prevenção aos setores da vigilância em saúde, aos ACS e aos ACE.

Em pesquisa realizada nos municípios que integram a $10^{\mathrm{a}}$ Regional de Saúde do Paraná, os enfermeiros relataram que não conseguem realizar educação em saúde devido à grande demanda curativa, falta de pessoal na equipe, trabalho burocrático e reuniões na Secretaria Municipal de Saúde, que tomam bastante tempo da rotina diária(24).

Conforme relatos de alguns profissionais sobre a realização de medidas preventivas somente após a ocorrência dos agravos, é importante ressaltar que a USF é um modelo de saúde que atua de forma integral e contínua, priorizando ações voltadas à prevenção, promoção e recuperação da saúde das pessoas, seja na unidade básica de saúde, no domicílio ou por meio de mobilização da comunidade ${ }^{(5)}$.

Desse modo, o tratamento e a cura não são mais o foco principal da assistência. Portanto, o enfermeiro, inserido neste ponto de atenção, deve realizar suas práticas, conforme os princípios e diretrizes estabelecidos. As medidas preventivas devem ser realizadas com frequência e não devem ser iniciadas apenas em decorrência de um aumento repentino no número de casos.

Frente ao exposto, cabe ressaltar que, no âmbito dos serviços de saúde, o enfermeiro é considerado um profissional dotado de conhecimentos, atitudes e habilidades voltados a promover uma assistência em saúde de forma integral e humanizada. Além de criar vínculos com a família e comunidade, o que possibilita a troca de saberes, diálogo e educação em saúde ${ }^{(25)}$.

Observou-se que um profissional mencionou realizar notificação dos casos de acidentes com escorpião. Este resultado é divergente do que se afirmou numa pesquisa realizada no município de Palmas-TO, na qual é preconizado que todo acidente causado por animal peçonhento atendido nas unidades de saúde deve ser notificado, mesmo que o paciente não tenha sido submetido à soroterapia. Acrescenta, ainda, que a ficha de investigação é um instrumento fundamental para conhecer o perfil do agravo, estabelecer protocolo de assistência ao paciente, programar ações para o controle dos animais e para distribuir soros, de acordo com a necessidade local ${ }^{(11)}$.

Diante de um cenário complexo, com as dificuldades de infraestrutura inerentes a um país em desenvolvimento, deve-se destacar a importância da intersetorialidade no combate aos escorpiões, em que o município tem a responsabilidade de implementar 
parcerias entre os órgãos envolvidos na limpeza urbana, vigilância em saúde, saneamento básico, educação, entre outros ${ }^{(4,19)}$.

Destaca-se como limitações deste estudo a consideração de apenas uma categoria profissional vinculada à equipe de saúde das unidades básicas, e a abordagem metodológica qualitativa, não direcionada a mensurar o conhecimento específico desses profissionais. Por isso, sugere-se que sejam desenvolvidas futuramente publicações com abordagem quantitativa para enfermeiros e outros profissionais da equipe de saúde.

\section{CONCLUSÃO}

A avaliação dos conhecimentos e práticas dos profissionais de enfermagem na APS acerca de acidentes escorpiônicos permitiu identificar que estes profissionais não desenvolvem uma assistência sistematizada, mas pontual, com base nos sinais e sintomas do quadro leve, e transferem ao serviço de referência a responsabilidade pelo atendimento ao caso, por desconhecimento das condutas estabelecidas para o acidentado. Além disso, a grande maioria não realiza ações educativas para a prevenção dos acidentes e repassam a responsabilidade ao sistema de vigilância em saúde.

Diante dos resultados, faz-se necessária a implementação de programas de treinamentos que abordem esse tema para os profissionais de enfermagem, com 0 intuito de melhorar a qualidade no cuidado ao acidentado, como também conduzir ações educativas à população, que reduzam a incidência de acidentes por escorpião.

Portanto, acredita-se que esse estudo possa contribuir para a implementação de estratégias que ampliem o cuidado às vítimas de escorpiões, além de propiciar maior visibilidade da temática para os profissionais que atuem na APS, sensibilizando-os a entenderem que a vítima acessará o serviço mais próximo e no qual mantém o vínculo e este deve ser resolutivo em sua demanda, sem negligenciar ações assertivas de saúde, nem transferir total responsabilidade a serviços de referência.

\section{REFERÊNCIAS}

1. Barbosa IR. Aspectos do escorpionismo no estado do Rio Grande do Norte. Rev. Saúde. Com. [Internet]. 2014 [acesso em 18 jul 2018]; 10(1). Disponível em: http://periodicos2.uesb.br/index.php/rsc/ article/view/281.

2. Kotviski BM, Barbola I de F. Aspectos espaciais do escorpionismo em Ponta Grossa, Paraná, Brasil. Cad. Saúde Pública. [Internet]. 2013 [acesso em 10 jul 2018]; 29(9). Disponível em: http://www.scielo.br/pdf/ csp/v29n9/a23v29n9.pdf.

3. Ministério da Saúde (BR). Acidentes por animais peçonhentos-escorpião. Situação epidemiológicadados. [Internet]. 2018 [acesso em 20 fev 2020]. Disponível em: https://www.saude.gov.br/saude-de-a-z/ acidentes-por-animais-peconhentos/13692-situacao-epidemiologica-dados.

4. Ministério da Saúde (BR). Secretaria de Vigilância em Saúde. Departamento de Vigilância Epidemiológica: manual de controle de escorpiões. [Internet]. Brasília: Ministério da Saúde; 2009 [acesso em 12 jul 2018]. Disponível em: http://bvsms.saude.gov.br/bvs/publicacoes/manual controle escorpioes. pdf.

5. Ministério da Saúde (BR). Portaria n. 2.436, de 21 de setembro de 2017. Aprova a Política Nacional de Atenção Básica, estabelecendo a revisão de diretrizes para a organização da Atenção Básica, no âmbito do Sistema Único de Saúde (SUS). [Internet]. 22 set 2017 [acesso em 14 jul 2018]. Disponível em: http:// bvsms.saude.gov.br/bvs/saudelegis/gm/2017/prt2436 2209 2017.html. 
6. Ministério da Saúde (BR). Fundação Nacional de Saúde. Manual de Diagnóstico e Tratamento de Acidentes por Animais Peçonhentos. [Internet]. Brasília: Ministério da Saúde; 2001 [acesso em 27 nov 2019]. Disponível em: http://portalarquivos2.saude.gov.br/images/pdf/2014/marco/14/Manual-deDiagnostico-e-Tratamento-de-Acidentes-por-Animais-Pe--onhentos.pdf.

7. Bardin L. Análise de Conteúdo. São Paulo: Edições 70; 2011.

8. Ministério da Saúde (BR). Conselho Nacional De Saúde. Diretrizes e normas regulamentadoras de pesquisa envolvendo seres humanos. Resolução n. 466, de 12 de dezembro de 2012. Brasília; 2012.

9. Mesquita FNB, Nunes MAP, Santana VR de, Machado Neto J, Almeida KBS de, Lima SO. Acidentes escorpiônicos no estado de Sergipe - Brasil. Rev. Fac. Ciênc. Méd. Sorocaba [Internet]. 2015 [acesso em 27 nov 2019]; 17(1). Disponível em: https://revistas.pucsp.br/RFCMS/article/view/19842/pdf.

10. Ciruffo PD, Coutinho L de O, Boroni JD, Diniz AET, Diniz WF. Escorpionismo: quadro clínico e manejo dos pacientes graves. Rev. méd. Minas Gerais. [Internet]. 2012 [acesso em 17 jul 2018]; 22(Suppl 8). Disponível em: http://rmmg.org/artigo/detalhes/648.

11. Parise EV. Vigilância e monitoramento dos acidentes por animais peçonhentos no município de Palmas, Tocantins, Brasil. Rev. Bras. Geogr. Méd. Saúde. [Internet]. 2016 [acesso em 20 jul 2018]; 12(22). Disponível em: http://www.seer.ufu.br/index.php/hygeia/article/viewFile/30701/18661.

12. Ministério da Saúde (BR). Animais Peçonhentos - Escorpiões. [Internet]. 2018 [acesso em 20 jul 2018]. Disponível em: http://portalms.saude.gov.br/saude-de-a-z/acidentes-por-animais-peconhentos-escorpiao.

13. Oliveira HFA de, Lopes YACF, Barros RM, Vieira AA, Leite R de S. Epidemiologia dos acidentes escorpiônicos ocorridos na Paraíba - Nordeste do Brasil. Rev Biol Farm. [Internet]. 2012 [acesso em 22 jul 2018]; 08(2). Disponível em: https://docplayer.com.br/40535681-Epidemiologia-dos-acidentesescorpionicos-ocorridos-na-paraiba-nordeste-do-brasil.html.

14. Pinto GFSG, Pessoa A de M, Silva Júnior NJ. Acidentes com escorpiões nas capitais Brasileiras entre 2007 e 2014. Estudos. [Internet]. 2015 [acesso em 24 jul 2018]; 42(4). Disponível em: http://seer.pucgoias. edu.br/index.php/estudos/article/viewFile/4369/2504.

15. Silva TMAS da, Teles DM, Braga PET, Aguiar FC de, Freire JE. Epidemiologia dos acidentes por escorpiões no Ceará no período de 2009 a 2012. Rev. Saúde. Com. [Internet]. 2015 [acesso em 25 jul 2018]; 11(3). Disponível em: http://periodicos2.uesb.br/index.php/rsc/article/view/371.

16. Melo MA de, Gouvêa EP, Odagima AM, Shitsuka DM, Shitsuka R. Escorpionismo: complicações, cuidados e prevenção. Revista Acadêmica da Faculdade Fernão Dias. [Internet]. 2017 [acesso em 25 jul 2018]; 4(14). Disponível em: http://www.fafe.edu.br/dados/pdf-uploads/245.pdf?1519311341252.

17. São Paulo. Secretaria de Estado da Saúde. Governo do Estado de São Paulo. Alerta aos Serviços de Saúde: acidente escorpiônico ou escorpionismo. [Internet]. 2018 [acesso em 24 nov 2019]. Disponível em: http://www.saude.sp.gov.br/resources/cve-centro-de-vigilancia-epidemiologica/areas-de-vigilancia/ doencas-de-transmissao-por-vetores-e-zoonoses/doc/peconhentos/escorpiao18 alerta profsaude.pdf.

18. Carvalho LAS, Pereira JMR. Aspectos Epidemiológicos de Acidentes Ofídicos no Município e Cuiabá, Mato Grosso. Connection Line. [Internet]. 2015 [acesso em 14 abr 2019]; 13. Disponível em: http:// periodicos.univag.com.br/index.php/CONNECTIONLINE/article/view/241.

19. Busato MA, Guarda C, Zulian V, Lutinski JA. Animais peçonhentos no ensino de Biologia: percepção de estudantes e professores de escolas públicas do oeste de Santa Catarina. Acta Scientiae. [Internet]. 2015 [acesso em 02 ago 2018]; 17(3). Disponível em: http://www.periodicos.ulbra.br/index.php/acta/ article/view/908/1316.

20. Ministério da Saúde (BR). Secretaria de Vigilância em Saúde. Departamento de Vigilância das Doenças Transmissíveis. Manual de vigilância, prevenção e controle de zoonoses: normas técnicas e operacionais. [Internet]. Brasília: Ministério da Saúde; 2016 [acesso em 04 ago 2018]. Disponível em: http://bvsms. saude.gov.br/bvs/publicacoes/manual vigilancia prevencao controle zoonoses.pdf. 
21. Ministério da Saúde (BR). Agentes Comunitários de Saúde e Agentes de Controle de Endemias. [Internet]. 2018 [acesso em 06 ago 2018]. Disponível em: https://www.saude.gov.br/trabalho-educacao-equalificacao/publicacoes/819-assuntos/trabalho-educacao-e-qualificacao/40727-agentes-comunitarios-desaude-e-agentes-de-controle-de-endemias.

22. Ministério da Saúde (BR). Secretaria de Vigilância em Saúde. Secretaria de Atenção à Saúde. Diretrizes Nacionais da Vigilância em Saúde. [Internet]. Brasília: Ministério da Saúde; 2010 [acesso em 28 jul 2019]. Disponível em: http://livroaberto.ibict.br/bitstream/1/901/1/diretrizes nacionais vigilancia saude.pdf.

23. Costa A de O, Alonzo HGA. Centros de Informação e Assistência Toxicológica no Brasil: descrição preliminar sobre sua organização e funções. Saúde debate. [Internet]. 2019 [acesso em 28 jul 2019]; 43(120). Disponível em: http://www.scielo.br/pdf/sdeb/v43n120/0103-1104-sdeb-43-120-0110.pdf.

24. Roecker S, Budó M de LD, Marcon SS. Trabalho educativo do enfermeiro na Estratégia Saúde da Família: dificuldades e perspectivas de mudança. Rev. esc. enferm. USP. [Internet]. 2012 [acesso em 08 ago 2018]; 46(3). Disponível em: http://dx.doi.org/10.1590/S0080-62342012000300016.

25. Souza KMJ, Seixas CT, David HMSL, Costa AQ da. Contribuições da Saúde Coletiva para o trabalho de enfermeiros. Rev bras enferm. [Internet]. 2017 [acesso em 27 nov 2019]; 70(3). Disponível em: http:// www.scielo.br/pdf/reben/v70n3/pt 0034-7167-reben-70-03-0543.pdf.

Recebido: 10/06/2019

Finalizado: 27/04/2020

Autor Correspondente:

Edjane Xavier dos Santos Silva

Centro de Saúde Dr. José Dustan Carvalho Soares

R. Santa Lúcia, 320 - 50731-430 - Recife, PE, Brasil

E-mail: janesantos.xavier@yahoo.com.br

Contribuição dos autores:

Contribuições substanciais para a concepção ou desenho do estudo; ou a aquisição, análise ou interpretação de dados do estudo - EXSS, JLCF

Elaboração e revisão crítica do conteúdo intelectual do estudo - WS, SRPA, DMS

Aprovação da versão final do estudo a ser publicado - DRSGT

Responsável por todos os aspectos do estudo, assegurando as questões de precisão ou integridade de qualquer parte do estudo - JAN 